\title{
VARIABILIDAD MORFOLÓGICA SEMINAL Y DEL VIGOR INICIAL DE GERMOPLASMA MEJORADO DE FRIJOL ${ }^{1}$
}

\author{
Raquel Celis-Velázquez², Cecilia Beatriz Peña-Valdivia², Mario Luna-Cavazos ${ }^{2}$, Juan Rogelio Aguirre-Rivera ${ }^{3}$, \\ Aquiles Carballo-Carballo², Carlos Trejo-López ${ }^{2}$
}

\begin{abstract}
RESUMEN
Variabilidad morfológica seminal y del vigor inicial de germoplasma mejorado de frijol. El objetivo del presente trabajo fue evaluar la variación en vigor seminal, basado en la morfología de la semilla y de las plántulas, del germoplasma mexicano de frijol (Phaseolus vulgaris L.) mejorado, para siembra comercial en diferentes zonas de México. Se utilizó el análisis multivariable de componentes principales y agrupamiento de las características morfológicas de la semillas (peso de la semilla, color, brillo, tono e intensidad del color de la testa, y proporción de cotiledones, testa y eje embrionario) y las plántulas (altura, biomasa de los cotiledones consumida en la germinación y emergencia, biomasa de folíolos, hipocótilo y raíz, diámetro de hipocótilo y longitud de hipocótilo y raíz) de 48 cultivares, sembrados a 2,5; 5,0 y $10,0 \mathrm{~cm}$ de profundidad. Fueron evaluadas en condiciones de invernadero, durante mayo y octubre de 2006, en Montecillo, Texcoco, México. Se realizaron análisis de varianza, comparación múltiple de medias y multivariables de ordenación y clasificación. El agrupamiento de los cultivares por su variabilidad de vigor inicial se basó en la capacidad para emerger. Las semillas más pesadas (38,88 g/100 semillas) generaron plántulas significativamente más vigorosas (mayor biomasa en raíz y folíolos $(0,20$ y $0,80 \mathrm{~g})$, altura $(24,24 \mathrm{~cm})$ y diámetro de hipocotilo $(0,43 \mathrm{~cm})$, respecto a las semillas pequeñas. El consumo de reservas para la emergencia fue similar en las tres profundidades $(90,3 \%)$. La variabilidad morfológica seminal de los 48 cultivares estudiados, fue amplia si se consideran caracteres como el peso de la semilla, del color (cromaticidad, luminosidad y tono) de la testa y razas; pero la variabilidad del vigor de los cultivares, dependiente de la profundidad de siembra, fue reducida.
\end{abstract}

Palabras clave: Phaseolus vulgaris L., plántula, profundidad de siembra, emergencia, razas.

\begin{abstract}
Variability of seed morphology and initial vigor of improved common bean germplasm. The objective was to evaluate seminal vigor variability, as a function of seed and seedling morphology of Mexican common bean (Phaseolus vulgaris L.) germplasm, improved for commercial sowing in different regions of Mexico. Seed morphology (weight of the seed, color, brightness, tone and color intensity of the seed coat, and proportion of cotyledons, seed coat and embryonic axis) and seedling morphology (height, biomass of cotyledons consumed in the germination and emergency, accumulated biomass in leaflets, hypocotyl and root, diameter and length of hypocotyl, and root length) of 48 cultivars, seeded at depths of $2.5,5.0$ and $10.0 \mathrm{~cm}$. Were evaluated under greenhouse conditions, during May and October of 2006, in Montecillo, Texcoco, Mexico. Analysis of variance, multiple mean comparison and multivariate analysis of ordination and classification were carried out. Grouping cultivars according to variability in initial vigor was based on the emergence capacity. Heavier seeds (38.88 g/100 seeds) produced seedlings significantly more vigorous, with more biomass in root and leaflets $(0.20$ and $0.80 \mathrm{~g})$, highest $(24.24 \mathrm{~cm})$ and rough hypocotyl $(0.43 \mathrm{~cm}$ in diameter) in comparison with the smallest seeds. Reserve utilization for seedling emergency was similar at all three depths (90.3\%). Variability in seminal morphology of 48 common bean improved cultivars was wide for characters such as the weight of the seed, parameters of the seed coat color (brightness, tone and intensity) and races, but the initial vigor variability of the 48 cultivars, depending on the depth of sowing, was reduced.
\end{abstract}

Key words: Phaseolus vulgaris L., seedling, deep sowing, emergence, races.

Recibido: 10 de diciembre, 2007. Aceptado: 25 de agosto, 2008.

2 Recursos Genéticos y Productividad, y Botánica. Colegio de Posgraduados. Carretera México-Texcoco km 35.5, Montecillo, Estado de México.56230.raquelcv@colpos.mx; cecilia@colpos.mx

3 Instituto de Investigaciones en Zonas Áridas, Universidad Autónoma de San Luís Potosí. Altaír 200. Col. del Llano. San Luís Potosí, S.L.P. iizd@uaslp.mx 


\section{INTRODUCCIÓN}

En México existen, del género Phaseolus, cerca de 65 especies, de las cuales sólo cinco fueron domesticadas en el periodo pre-colombino: Phaseolus acutifolius, $P$. coccineus, $P$. lunatus, $P$. dumosus y $P$. vulgaris L. (Kaplan y Lynch 1999). Entre estas especies, $P$. vulgaris (frijol) es la más importante, pues su cultivo ocupa más del $85 \%$ de la superficie sembrada de todas las especies de Phaseolus en el mundo (Singh, 2001). Mundialmente se siembran 25 millones de hectáreas con frijol y México es uno de los principales productores de esta leguminosa, con 2,2 millones de hectáreas sembradas anualmente, con una producción de 1,2 millones de toneladas y rendimiento promedio de $643 \mathrm{~kg} / \mathrm{ha}$ (Cruz 2006). Actualmente, se acepta la existencia de tres acervos genéticos en América, el mesoamericano, el andino del norte y el andino del sur (Debouck et al. 1993); a la vez, los acervos o centros de frijol domesticado mesoamericano y del sur de los andes fueron subdivididos en tres razas cada uno (i.e. Durango, Mesoamérica y Jalisco para el primero, y Chile, Nueva Granada, y Perú para el segundo), que incluyen diferentes variantes cultivadas como consecuencia de la domesticación. Cada raza tiene características, adaptación ecológica y respuesta agronómica propia (Beebe et al. 2000).

En México, desde hace más de 50 años se ha trabajado en mejoramiento genético de frijol, el cual ha permitido la obtención de más de 120 cultivares mejorados, para diferentes regiones productoras, y la base de este programa es el gran acervo de cultivares criollos dispersos en todo el territorio (Voysest 2000, Vargas et al. 2006). Sin embargo, se ha señalado que la aceptación de los cultivares mejorados por los productores es limitada y que, en cierta forma, continúan predominando los cultivares criollos, pues en su región de origen frecuentemente generan rendimientos altos y son preferidos por los consumidores locales (Rosales et al. 2003). En contraste, en las áreas de mayor producción, se cultivan grandes extensiones con un número reducido de cultivares mejorados y criollos, los que dominan el mercado nacional y cubren la demanda de las poblaciones urbanas mayores; situación que tiende a disminuir la diversidad genética (Peña-Valdivia y Aguirre 2007, Vargas et al. 2006). El reconocimiento de dos grupos mayores de germoplasma de frijol (mesoamericano y andino) ha ayudado a confirmar su participación en la selección y desarrollo de los cultivares mejorados que dominan la preferencia de los agricultores empresariales y consumidores (Singh 1999, Voysest 2000). El conocimiento de la diversidad genética puede ayudar a generar acciones para su conservación, uso eficiente y mejoramiento (Singh et al. 1991). Este conocimiento incluye las características morfológicas, fenológicas, bioquímicas, genéticas y otras, en el nivel de la semilla y de la planta (Rosales et al. 2003, Singh et al. 1991, Voysest 2000).

El vigor de la plántula es cuantificado usualmente como el peso o tamaño de las plantas jóvenes que aún dependen de las reservas del endospermo (etapa heterótrofa). Luego, al ser consumidas las reservas del endospermo, las plántulas dependen de su capacidad para generar asimilados, formar un dosel y superar a las arvenses (Hamman et al. 2002). La emergencia es dependiente de la calidad de la semilla y del ambiente de cultivo (humedad del suelo, patógenos, temperatura e impedancia, como los más importantes). Unas semanas después de la emergencia, las diferencias morfológicas entre las plántulas permitirán conocer el denominado vigor temprano, y si éste es pobre, el cultivo no podrá competir con las arvenses (Hamman et al. 2002, Revilla et al. 1999). Por ello, resulta de interés conocer la variación en el vigor temprano del germoplasma mejorado de frijol, dada su importancia antropocéntrica.

Los métodos multivariables se utilizan también en la caracterización de los recursos fitogenéticos, pues permiten el análisis simultáneo de numerosos individuos, cultivares, poblaciones, etc. y de un número amplio de características. Entre estos métodos, el análisis de componentes principales (ACP), el factorial de correspondencias (MCA) y el de conglomerados, son herramientas adecuadas para analizar los datos que se generan de la caracterización y evaluación preliminar de germoplasma, pues permiten conocer la relación entre las características cuantitativas y muestran las semejanzas y contrastes entre las muestras estudiadas; además, permiten seleccionar las variables cuantitativas discriminantes entre las unidades de estudio (Hidalgo 2003). En efecto, Núñez y Barrientos (2006) propusieron el uso de $\mathrm{ACP}$ como una herramienta para describir la variación interna de grupos o poblaciones en dos o tres combinaciones lineales de variables agrupadas, y observar la dispersión de los individuos en 
planos con dimensionalidad reducida. Así, Vidal-Barahona et al. (2006) establecieron el nivel de semejanza de 21 genotipos de frijol, con base en características morfológicas y moleculares mediante ACP. Beebe et al. (2000) determinaron las semejanzas genéticas en una muestra de 269 cultivares tradicionales de frijol de América Central, mediante MCA. Rosales et al. (2003) determinaron la diversidad genética de 120 cultivares de frijol mexicano mejorado para siembra comercial en diferentes zonas productoras, con base en 72 características morfológicas y cinco indicadores polimórficos para intersecuencias simples repetidas, mediante ACP y conglomerados; mientras, Otálora et al. (2006) evaluaron las características agronómicas, químicas y de cocción del grano de frijol, y buscaron correlacionar y seleccionar las variables de mayor incidencia en la caracterización de 29 cultivares de frijol.

El objetivo de este trabajo fue evaluar la variabilidad del vigor inicial, con base en la morfología de la semilla y de las plántulas del germoplasma de frijol mejorado para las diferentes zonas productoras de México. Para lograr este objetivo, se realizaron análisis multivariables de componentes principales y agrupamiento aglomerativos basados en las características morfológicas de las semillas y las plántulas de 48 cultivares mejorados fitogenéticamente, sembrados a 2,5, 5,0 y $10 \mathrm{~cm}$ de profundidad. La hipótesis planteada es que el mejoramiento de los cultivares ha tendido a incrementar la homogeneidad morfológica tanto de su semilla como de sus plántulas y por lo tanto de su vigor inicial.

\section{MATERIALES Y MÉTODOS}

\section{Material biológico}

En el presente estudio se incluyeron 48 cultivares de frijol (Phaseolus vulgaris L.) mejorado, desarrollados en el Programa de Frijol del Instituto Nacional de Investigaciones Forestales, Agrícolas y Pecuarias (INIFAP), México, para su siembra comercial en las diferentes zonas agrícolas de México (Cuadro 1). Las semillas fueron multiplicadas en el campo experimental del Colegio de Postgraduados, en el ciclo primavera-verano de 2006; después de ser cosechadas,
Cuadro 1. Cultivares de frijol (Phaseolus vulgaris L.) utilizados en la evaluación de la variabilidad morfológica seminal y vigor inicial. Texcoco, México. 2006.

\begin{tabular}{|c|c|c|c|c|c|}
\hline Núm. & Cultivar & Raza & Núm. & Cultivar & Raza* \\
\hline 1 & Amarillo 154 & $\mathrm{~J}$ & 25 & Negro 8025 & MA \\
\hline 2 & $\begin{array}{l}\text { Azufrado Hi- } \\
\text { guera }\end{array}$ & NG & 26 & Negro Altiplano & $\mathrm{D}$ \\
\hline 3 & $\begin{array}{l}\text { Azufrado Na- } \\
\text { miquipa }\end{array}$ & $\mathrm{D} / \mathrm{J}$ & 27 & Negro Chapingo & _ \\
\hline 4 & $\begin{array}{l}\text { Azufrado No- } \\
\text { roeste }\end{array}$ & NG & 28 & Negro Cotaxtla & MA \\
\hline 5 & $\begin{array}{l}\text { Azufrado Pi- } \\
\text { mono }\end{array}$ & NG & 29 & Negro Durango & $\mathrm{D}$ \\
\hline 6 & $\begin{array}{l}\text { Azufrado Re- } \\
\text { gional }\end{array}$ & NG & 30 & $\begin{array}{l}\text { Negro Huaste- } \\
\text { co } 81\end{array}$ & MA \\
\hline 7 & $\begin{array}{l}\text { Azufrado Ta- } \\
\text { patío }\end{array}$ & $\mathrm{D} / \mathrm{J}$ & 31 & Negro INIFAP & MA \\
\hline 8 & Bayo INIFAP & $\mathrm{J}$ & 32 & Negro Jamapa & MA \\
\hline 9 & Bayo Madero & D & 33 & Negro Medellín & MA \\
\hline 10 & $\begin{array}{l}\text { Bayo Mecen- } \\
\text { tral }\end{array}$ & $\mathrm{J}$ & 34 & Negro Nayarit & MA \\
\hline 11 & Bayo Victoria & D & 35 & Negro Otomí & $\mathrm{J}$ \\
\hline 12 & $\begin{array}{l}\text { Bayo Zaca- } \\
\text { tecas }\end{array}$ & D & 36 & Negro Pacífico & MA \\
\hline 13 & $\begin{array}{l}\text { Bayo Zacate- } \\
\text { cas } 20\end{array}$ & D & 37 & Negro Perla & MA \\
\hline 14 & Bayomex & NG & 38 & $\begin{array}{l}\text { Negro Sahua- } \\
\text { toba }\end{array}$ & MA \\
\hline 15 & Delicias 72 & $\mathrm{D}$ & 39 & Negro Sinaloa & MA \\
\hline 16 & $\begin{array}{l}\text { Flor de Du- } \\
\text { razno }\end{array}$ & NG & 40 & Negro Tacaná & MA \\
\hline 17 & $\begin{array}{l}\text { Flor de Mayo } \\
\text { Sol }\end{array}$ & $\mathrm{J}$ & 41 & Negro Tropical & _ \\
\hline 18 & $\begin{array}{l}\text { Flor de Mayo } \\
2000\end{array}$ & $\mathrm{~J}$ & 42 & Negro Vizcaya & - \\
\hline 19 & FMC & $\mathrm{J}$ & 43 & Negro Zacatecas & $\mathrm{J}$ \\
\hline 20 & $\begin{array}{l}\text { Flor de Mayo } \\
\text { M38-2002 }\end{array}$ & $\mathrm{J}$ & 44 & Ojo de Cabra & $\mathrm{D}$ \\
\hline 21 & $\begin{array}{l}\text { Flor de Mayo } \\
\text { M } 38\end{array}$ & $\mathrm{~J}$ & 45 & Pinto Anzalduas & $\mathrm{D} / \mathrm{J}$ \\
\hline 22 & $\begin{array}{l}\text { Garbancillo } \\
\text { Supremo }\end{array}$ & $\mathrm{J}$ & 46 & Pinto Bayacora & $\mathrm{D}$ \\
\hline 23 & Manzano & $\mathrm{D} / \mathrm{J}$ & 47 & Pinto Mestizo & $\mathrm{D}$ \\
\hline 24 & Negro 150 & $\mathrm{~J}$ & 48 & Pinto Villa & $\mathrm{D}$ \\
\hline
\end{tabular}

* Razas: $\mathrm{D}=$ Durango, $\mathrm{J}=$ Jalisco, MA= Mesoamericana y NG= Nueva Granada.

las semillas fueron almacenadas a $18 \pm 1{ }^{\circ} \mathrm{C}$ a una H.R. entre 60 y $70 \%$ durante unas semanas, hasta su evaluación. 


\section{Selección y siembra de las semillas}

Para cada cultivar se tomaron 1.000 semillas al azar, de lotes de tamaño variable cosechados en el ciclo agrícola previo al estudio; luego, estas muestras se separaron en semilla chica, mediana y grande, con cribas del número 4,0; 4,65 ó 5,0 en el caso de los cultivares con semillas chicas, y del número 6 y 6,5 para las demás. De cada cultivar se escogieron 20 semillas de tamaño mediano, por triplicado, se pesaron individualmente y se sembraron en macetas de 201 de capacidad, con una mezcla de tierra de monte y arena $(2: 1)$ como sustrato, a tres profundidades 2,$5 ; 5,0$ y 10,0 $\mathrm{cm}$, y se mantuvieron en un invernadero del Colegio de Postgraduados, en Montecillo, Texcoco, México, a partir de mayo de 2006. Se aplicó un riego a saturación en la siembra y después, cada tercer día o según se requiriera para mantener el substrato húmedo.

\section{Variables evaluadas}

Se determinó el peso hectolítrico de 10 grupos de 100 semillas de cada cultivar, en una balanza analítica Scientech (Mod. SA100; EE.UU.) (precisión de 0,0001 g). Se determinaron tres variables del color de la semilla (luminosidad o brillo, matiz o tono e índice de saturación o intensidad del color) con un colorímetro Hunter Lab (D25PC2). Se registró el peso de las estructuras (cotiledón, testa y eje embrionario) secos de 25 semillas, separados manual e individualmente, con ayuda de un bisturí, para ello se usó una balanza analítica (precisión de 0,0001 g), y una estufa LAB-LINE (L-C OVENS); los materiales fueron deshidratados a $75^{\circ} \mathrm{C}$, por cuatro días, antes de pesarse, y se calculó su proporción en las semillas.

Cuando las plántulas expusieron la primera hoja trifoliolada se midió su altura $(\mathrm{cm})$ y el diámetro $(\mathrm{cm})$ del hipocótilo, enseguida fueron cosechadas y se midió la longitud de su raíz $(\mathrm{cm})$, luego fueron disecadas y sus estructuras se mantuvieron en una estufa a $75^{\circ} \mathrm{C}$ durante 72 horas. Al final de este tiempo se determinó el peso (g) de la raíz, folíolos, hipocótilo y cotiledones. El porcentaje de reservas consumidas fue calculado a partir del promedio de la materia seca de los cotiledones de la semilla de cada cultivar y el peso deshidratado de los cotiledones en las plántulas.

\section{Diseño experimental y análisis estadístico de los resultados}

Se utilizó un modelo experimental completamente al azar con tres repeticiones; cada repetición estuvo constituida por 20 semillas, como unidad experimental. Los resultados fueron ordenados con el análisis multivariable de componentes principales (ACP) para establecer la dispersión de los cultivares en planos con dimensionalidad reducida y agrupados para clasificar los cultivares en dependencia de su homogeneidad (Franco e Hidalgo 2003). En ambos casos, la variación entre los individuos, en las variables evaluadas, fue representada gráficamente, con la dimensionalidad reducida por agrupamiento de todas las variables en dos o tres combinaciones lineales con características óptimas para la representación subyacente (Núñez y Barrientos 2006).

Con la finalidad de reconocer la variabilidad morfológica seminal entre los 48 cultivares, independiente del vigor, se realizó el análisis de varianza y comparación múltiple de medias para las ocho variables de las semillas; luego, para evaluar la variabilidad del vigor inicial se realizó un segundo grupo de análisis multivariable que incluyó sólo cuatro características de la semilla (proporción de cotiledones, eje embrionario, testa en la semillas y tamaño de semilla o peso de 100 semillas) y las ocho de las plántulas (altura, diámetro del hipocótilo, longitud del hipocótilo, longitud de la raíz, peso de la raíz, folíolos, hipocótilo y cotiledones).

\section{RESULTADOS Y DISCUSIÓN}

El color de la semilla de frijol es una característica que identifica la preferencia de los consumidores regionalmente. En este estudio se incluyó una gama amplia de colores entre los 48 cultivares; sin embargo, la evaluación formal del color y la comparación múltiple de medias de sus parámetros demostró que el tono (atributo de la sensación visual según la cual una superficie parece similar a uno, o a proporciones de dos de los colores percibidos amarillo, naranja, rojo, verde, azul y púrpura) fue similar (entre 39,71 y $91,17^{\circ}$ ) en poco más de $60 \%$ del grupo de cultivares y 
los valores de tono estadísticamente mayores $(301,2$ a $\left.329,8^{\circ}\right)$ correspondió sólo a un grupo pequeño (13\%) del total de los cultivares. Además, $40 \%$ de la muestra fue homogénea (entre 0,33 y $1,10^{\circ}$ ) en la cromaticidad (pureza relativa del color dominante), al conformarse únicamente de frijoles con testa negra. En contraste, sólo un grupo pequeño (17\%) de cultivares presentó luminosidad (atributo de la sensación visual según la cual una superficie emite más o menos luz, lo cual permite calificar un tono como oscuro o claro) estadísticamente mayor $\left(64,21\right.$ a $\left.67,25^{\circ}\right)$ (Cuadro 2). Las proporciones elevadas de semillas con tono y cromaticidad de testa estadísticamente similares indican que la diversidad de color en la muestra evaluada es reducida. Resultados similares de reducción en la diversidad genética fue documentada por Gepts et al. (1986) en faseolina, y por Chacón et al. (2005), en secuencias de nucleótidos en cloroplastos.

Los cultivares con los contenidos significativamente mayores de cotiledón presentaron los contenidos menores de testa, y viceversa. En contraste, sobresalió la homogeneidad de la proporción del eje embrionario en las semillas; así, con excepción del cv. Negro Perla, con una proporción significativamente alta de eje embrionario $(4,06 \%)$ los cultivares estudiados son estadísticamente similares en la proporción de este órgano en sus semillas (1,23 a 2,00 \%) (Cuadro 2). Estos resultados contrastan con la proporción y su variabilidad de las estructuras en las semillas de frijol silvestre. En efecto, Flores et al. (2002) encontró en semillas de cinco variantes de frijol silvestre, originarias de Chihuahua, Durango y Tlaxcala, México, que el peso seco de la testa representó entre el 11,8 y el $20,4 \%$, y el del eje embrionario entre el 1,5 y $4,5 \%$ del peso total de la semilla, pero en una variante silvestre originaria de Oaxaca, México, con semilla significativamente más grande que las otras cinco $(14,9 \mathrm{~g} / 100$ semillas contra 6,34 a 13,68 , respectivamente), tuvo proporciones de testa $(9,9 \%)$ y eje embrionario $(1,5 \%)$ semejantes a las semillas de variantes domesticadas (de 7,1 a 9,9 y de 0,69 a $1,7 \%$, respectivamente).

Peña-Valdivia et al. (1998) demostraron la existencia de correlaciones positivas y altamente significativas entre el peso de los cotiledones, el peso del eje embrionario y el de la testa de las semillas de frijol domesticado; con base en sus resultados, estos autores señalaron que con el tamaño de la semilla se modifica proporcionalmente cada una de sus estructuras anatómicas pero no se altera su papel en la viabilidad ni en la germinación. Así mismo, con base en evidencias experimentales, Peña-Valdivia et al. (1998) postularon que con el incremento del tamaño de la semilla, resultado de la domesticación, aumentó el peso absoluto de la testa, pero a la vez su peso relativo disminuyó alrededor del $50 \%$.

El peso volumétrico es una característica de calidad de la semilla. Cultivares con valores iguales o superiores a $75 \mathrm{~kg} / \mathrm{hl}$, según la norma del Servicio Nacional de Inspección y Certificación de Semillas, de la Secretaría de Agricultura, Ganadería y Desarrollo Rural (Moreno 1996), son semillas que germinarán y desarrollarán una plántula sana. Los resultados del Cuadro 2 muestran que de los 48 cultivares estudiados 42 poseen semilla con peso superior al de la norma, y dos, Delicias 72 (Núm. 15) y Ojo de Cabra (Núm. 44) poseen semilla menor que la norma mexicana.

El establecimiento de la plántula depende, en gran parte, de las características fisiológicas y bioquímicas de la semilla, que determinan su eficiencia en el uso de sus reservas para iniciar y sostener el crecimiento de la plántula, antes de que ésta se transforme en un organismo autótrofo (Soltani et al. 2006). En general, el frijol después de la emergencia tarda varias semanas en desarrollar un dosel suficiente para evitar el establecimiento de las arvenses, las cuales, en contraste, regularmente son más rápidas y eficientes para formar área foliar. Se ha señalado que la emergencia y establecimiento pobres de los cultivos es un problema común que podría resolverse, al menos parcialmente, con la identificación de cultivares con alto vigor de emergencia (Nabi et al. 2001). La caracterización fisiológica de la emergencia y establecimiento de la planta en dependencia de la profundidad de siembra permitiría reconocer diferencias entre cultivares de frijol en caracteres como rapidez y homogeneidad de emergencia, tasa de crecimiento de la plántula, exposición y desarrollo del follaje.

\section{Análisis de componentes principales de las carac- terísticas morfológicas de semillas y plántulas}

Los análisis multivariables incluyeron cuatro características de las semillas (peso de 100 semillas, proporción de cotiledones, testa y eje embrionario) y 
Cuadro 2. Características morfológicas y de color de la semilla de 48 cultivares de frijol mejorado en México para diferentes regiones agrícolas. Montecillo, Texcoco, México. 2006.

\begin{tabular}{|c|c|c|c|c|c|c|c|c|c|c|c|c|}
\hline \multirow{2}{*}{$\begin{array}{l}\text { Nú- } \\
\text { mero }\end{array}$} & \multirow[t]{2}{*}{ Cultivar } & \multicolumn{4}{|c|}{ Color } & \multicolumn{2}{|c|}{ Peso } & \multirow{2}{*}{$\begin{array}{l}\text { Cotile- } \\
\text { dón }(\%)\end{array}$} & \multirow{2}{*}{$\begin{array}{c}\text { Testa } \\
(\%)\end{array}$} & \multirow{2}{*}{$\begin{array}{l}\mathbf{E , E}, \\
(\%)\end{array}$} & \multirow{2}{*}{$\begin{array}{l}\text { Cotile- } \\
\text { dón/Tes- } \\
\text { ta }\end{array}$} & \multirow{2}{*}{$\begin{array}{l}\text { Coti- } \\
\text { ledón/ } \\
\text { E,E, }\end{array}$} \\
\hline & & & $\begin{array}{l}\text { Lumino- } \\
\text { sidad }\left({ }^{\circ}\right)\end{array}$ & $\begin{array}{l}\text { Cromati- } \\
\text { cidad }\left({ }^{\circ}\right)\end{array}$ & Tono $\left({ }^{\circ}\right)$ & $\begin{array}{c}\text { Volu- } \\
\text { métrico } \\
(\mathrm{kg} / \mathrm{hl})\end{array}$ & $\begin{array}{c}100 \\
\text { semillas } \\
(\mathrm{g})\end{array}$ & & & & & \\
\hline 1 & $\begin{array}{l}\text { Amarillo } \\
154\end{array}$ & $\begin{array}{l}\text { Amarillo } \\
\text { mostaza }\end{array}$ & 47,99 h-k & $46,52 \mathrm{a}$ & $67,96 \mathrm{j}-\mathrm{m}$ & $78 \mathrm{~m}$ & $24,65 \mathrm{pq}$ & $89,21 \mathrm{~b}-\mathrm{g}$ & 9,18 a-h & $1,75 \mathrm{~b}$ & $10,55 \mathrm{c}-\mathrm{f}$ & $53,4 \mathrm{ab}$ \\
\hline 2 & $\begin{array}{l}\text { Azufrado } \\
\text { Higuera }\end{array}$ & $\begin{array}{l}\text { Amarillo } \\
\text { azufrado }\end{array}$ & $65,41 \mathrm{ab}$ & $41,54 \mathrm{ab}$ & $87,71 \mathrm{jk}$ & $235 \mathrm{j}$ & $51,57 \mathrm{a}$ & $92,49 \mathrm{a}$ & $6,14 \mathrm{i}$ & $1,35 \mathrm{~b}$ & $15,45 \mathrm{a}$ & $73,57 \mathrm{ab}$ \\
\hline 3 & $\begin{array}{l}\text { Azufrado } \\
\text { Namiquipa }\end{array}$ & $\begin{array}{l}\text { Amarillo } \\
\text { grisáceo }\end{array}$ & $61,14 \mathrm{~d}$ & $27,71 \mathrm{~d}-\mathrm{g}$ & $75,89 \mathrm{j}-\mathrm{m}$ & $260 \mathrm{e}$ & $29,93 \mathrm{~m}$ & 91,22 a-d & $7,40 \mathrm{f}-\mathrm{i}$ & $1,43 \mathrm{~b}$ & 12,97 a-e & $102,43 \mathrm{ab}$ \\
\hline 4 & $\begin{array}{l}\text { Azufrado } \\
\text { Noroeste }\end{array}$ & $\begin{array}{l}\text { Amarillo } \\
\text { claro }\end{array}$ & 65,06 a-c & $32,91 \mathrm{de}$ & 84,16 j-1 & $230 \mathrm{k}$ & $46,27 \mathrm{~b}$ & 91,43 a-c & $7,28 \mathrm{~g}-\mathrm{i}$ & $1,60 \mathrm{~b}$ & 12,59 a-e & $66,5 \mathrm{ab}$ \\
\hline 5 & $\begin{array}{l}\text { Azufrado } \\
\text { Pimono }\end{array}$ & $\begin{array}{l}\text { Amarillo } \\
\text { claro }\end{array}$ & $65,40 \mathrm{ab}$ & $29,10 \mathrm{~d}-\mathrm{g}$ & 82,95 j-1 & 2251 & $37,05 \mathrm{~h}$ & $91,91 \mathrm{ab}$ & 6,76 hi & $1,53 \mathrm{~b}$ & 13,87 a-c & $67,04 \mathrm{ab}$ \\
\hline 6 & $\begin{array}{l}\text { Azufrado } \\
\text { Regional }\end{array}$ & $\begin{array}{l}\text { Amarillo } \\
\text { claro }\end{array}$ & $67,45 \mathrm{a}$ & $34,22 \mathrm{c}-\mathrm{e}$ & $84,16 \mathrm{j}-1$ & $235 \mathrm{j}$ & $34,99 \mathrm{i}$ & 89,36 b-g & $9,45 \mathrm{a}-\mathrm{g}$ & $1,23 \mathrm{~b}$ & $10,43 \mathrm{c}-\mathrm{f}$ & $107,69 \mathrm{a}$ \\
\hline 7 & $\begin{array}{l}\text { Azufrado } \\
\text { Tapatío }\end{array}$ & $\begin{array}{l}\text { Amarillo } \\
\text { grisáceo }\end{array}$ & $66,23 \mathrm{a}$ & $27,66 \mathrm{~d}-\mathrm{g}$ & $83,34 \mathrm{j}-1$ & $250 \mathrm{~g}$ & 31,081 & 90,62 a-f & $7,77 \mathrm{c}-\mathrm{i}$ & $1,65 \mathrm{~b}$ & 12,11 a-f & $99,39 \mathrm{ab}$ \\
\hline 8 & $\begin{array}{l}\text { Bayo INI- } \\
\text { FAP }\end{array}$ & Amarillo & $67,03 \mathrm{a}$ & $42,50 \mathrm{a}$ & $60,81 \mathrm{j}-\mathrm{m}$ & $250 \mathrm{~g}$ & $27,81 \mathrm{n}$ & 90,98 a-f & $7,28 \mathrm{~g}-\mathrm{i}$ & $1,92 \mathrm{~b}$ & 12,99 a-d & $57,13 \mathrm{ab}$ \\
\hline 9 & $\begin{array}{l}\text { Bayo Ma- } \\
\text { dero }\end{array}$ & Amarillo & $54,32 \mathrm{ef}$ & $41,16 \mathrm{a}-\mathrm{c}$ & $50,46 \mathrm{k}-\mathrm{m}$ & $270 \mathrm{c}$ & $37,00 \mathrm{~h}$ & 89,63 a-g & 9,10 a-h & $1,36 \mathrm{~b}$ & $9,93 \mathrm{c}-\mathrm{f}$ & $69,27 \mathrm{ab}$ \\
\hline 10 & $\begin{array}{l}\text { Bayo Me- } \\
\text { central }\end{array}$ & $\begin{array}{l}\text { Amarillo } \\
\text { pajizo }\end{array}$ & $64,21 \mathrm{a}-\mathrm{d}$ & $26,96 \mathrm{e}-\mathrm{h}$ & $73,04 \mathrm{j}-\mathrm{m}$ & $250 \mathrm{~g}$ & $27,22 \mathrm{n}$ & $90,32 \mathrm{a}-\mathrm{g}$ & 8,03 b-i & $1,75 \mathrm{~b}$ & 11,66 a-f & $56,16 \mathrm{ab}$ \\
\hline 11 & $\begin{array}{l}\text { Bayo Vic- } \\
\text { toria }\end{array}$ & Amarillo & $51,06 \mathrm{f}-\mathrm{h}$ & $31,01 \mathrm{~d}-\mathrm{f}$ & $58,16 \mathrm{j}-\mathrm{m}$ & $290 \mathrm{a}$ & $46,89 \mathrm{~b}$ & 89,43 b-g & 9,14 a-h & $1,37 \mathrm{~b}$ & $10,12 \mathrm{c}-\mathrm{f}$ & $70,57 \mathrm{ab}$ \\
\hline 12 & $\begin{array}{l}\text { Bayo Zaca- } \\
\text { tecas }\end{array}$ & Amarillo & $54,96 \mathrm{e}$ & 30,82 d-f & $62,20 \mathrm{j}-\mathrm{m}$ & $270 \mathrm{c}$ & $39,07 \mathrm{~g}$ & $88,56 \mathrm{c}-\mathrm{g}$ & 9,99 a-e & $1,43 \mathrm{~b}$ & $9,44 \mathrm{c}-\mathrm{f}$ & $71,41 \mathrm{ab}$ \\
\hline 13 & $\begin{array}{l}\text { Bayo Zaca- } \\
\text { tecas } 20\end{array}$ & Amarillo & $62,14 \mathrm{~b}-\mathrm{d}$ & $28,81 \mathrm{~d}-\mathrm{g}$ & $65,77 \mathrm{j}-\mathrm{m}$ & $265 \mathrm{~d}$ & $41,16 \mathrm{e}$ & $88,80 \mathrm{c}-\mathrm{g}$ & $9,62 \mathrm{a}-\mathrm{g}$ & $1,46 \mathrm{~b}$ & $9,34 \mathrm{~d}-\mathrm{f}$ & $64,85 \mathrm{ab}$ \\
\hline 15 & Delicias 72 & $\begin{array}{l}\text { Castaño } \\
\text { claro }\end{array}$ & $44,45 \mathrm{~lm}$ & $23,78 \mathrm{f}-\mathrm{k}$ & $56,08 \mathrm{j}-\mathrm{m}$ & 69 o & $18,28 \mathrm{v}$ & $88,85 \mathrm{c}-\mathrm{g}$ & 9,88 a-f & $1,25 \mathrm{~b}$ & $9,35 \mathrm{~d}-\mathrm{f}$ & $90,60 \mathrm{ab}$ \\
\hline 16 & $\begin{array}{l}\text { Flor de Du- } \\
\text { razno }\end{array}$ & $\begin{array}{l}\text { Rosado/fon- } \\
\text { do pajizo }\end{array}$ & $\begin{array}{l}46,45 \\
k-m\end{array}$ & $17,59 \mathrm{j}-\mathrm{m}$ & $44,36 \mathrm{~lm}$ & $260 \mathrm{e}$ & $43,6 \mathrm{~d}$ & 90,62 a-f & $7,79 \mathrm{c}-\mathrm{i}$ & $1,54 \mathrm{~b}$ & 11,89 a-f & $65,10 \mathrm{ab}$ \\
\hline 17 & $\begin{array}{l}\text { Flor de } \\
\text { Mayo Sol }\end{array}$ & $\begin{array}{l}\text { Rosado/fon- } \\
\text { do pajizo }\end{array}$ & $50,40 \mathrm{~g}-\mathrm{j}$ & $18,92 \mathrm{i}-\mathrm{m}$ & $44,74 \mathrm{~lm}$ & $255 \mathrm{f}$ & $24,32 \mathrm{pq}$ & 89,36 b-g & 8,87 a-h & $1,66 \mathrm{~b}$ & $10,77 \mathrm{~b}-\mathrm{f}$ & $56,29 \mathrm{ab}$ \\
\hline 18 & $\begin{array}{l}\text { Flor de } \\
\text { Mayo } 2000\end{array}$ & $\begin{array}{l}\text { Rosado/fon- } \\
\text { do pajizo }\end{array}$ & $50,89 \mathrm{f}-\mathrm{i}$ & $14,97 \mathrm{~lm}$ & $39,71 \mathrm{~m}$ & $255 \mathrm{f}$ & $24,71 \mathrm{p}$ & 89,38 b-g & 8,78 a-h & $1,44 \mathrm{~b}$ & $10,73 \mathrm{c}-\mathrm{f}$ & $65,35 \mathrm{ab}$ \\
\hline 19 & FMC & $\begin{array}{l}\text { Rosado/fon- } \\
\text { do pajizo }\end{array}$ & $\begin{array}{l}46,14 \\
k-m\end{array}$ & $\begin{array}{l}19,85 \\
\mathrm{H}-\mathrm{m}\end{array}$ & $43,65 \mathrm{~lm}$ & $255 \mathrm{f}$ & $29,48 \mathrm{~m}$ & $88,66 \mathrm{c}-\mathrm{g}$ & 9,47 a-g & $1,72 \mathrm{~b}$ & $9,74 \mathrm{c}-\mathrm{f}$ & $57,13 \mathrm{ab}$ \\
\hline 20 & $\begin{array}{l}\text { Flor de } \\
\text { Mayo M38- } \\
2002\end{array}$ & $\begin{array}{l}\text { Rosado/fon- } \\
\text { do pajizo }\end{array}$ & 48,20 h-k & $\begin{array}{l}17,37 \quad \mathrm{k}- \\
\mathrm{m}\end{array}$ & $44,37 \mathrm{~lm}$ & $255 \mathrm{f}$ & $33,11 \mathrm{j}$ & $88,57 \mathrm{c}-\mathrm{g}$ & 9,81 a-f & $1,52 \mathrm{~b}$ & $9,115 \mathrm{~d}-\mathrm{f}$ & $68,96 \mathrm{ab}$ \\
\hline 21 & $\begin{array}{l}\text { Flor de } \\
\text { Mayo M } 38\end{array}$ & $\begin{array}{l}\text { Rosado/fon- } \\
\text { do pajizo }\end{array}$ & $50,28 \mathrm{~g}-\mathrm{j}$ & $19,33 \mathrm{i}-\mathrm{m}$ & $48,29 \mathrm{k}-\mathrm{m}$ & $245 \mathrm{~h}$ & $23,93 \mathrm{qr}$ & 89,30 b-g & 9,03 a-h & $1,51 \mathrm{~b}$ & $10,27 \mathrm{c}-\mathrm{f}$ & $65,89 \mathrm{ab}$ \\
\hline 22 & $\begin{array}{l}\text { Garbancillo } \\
\text { Supremo }\end{array}$ & Amarillo & $61,87 \mathrm{c}-\mathrm{d}$ & 29,66 d-f & 78,77 j-m & $245 \mathrm{~h}$ & $21,51 \mathrm{~s}$ & 89,95 a-g & $7,76 \mathrm{c}-\mathrm{i}$ & $1,60 \mathrm{~b}$ & $12,28 \mathrm{a}-\mathrm{f}$ & $58,83 \mathrm{ab}$ \\
\hline 23 & Manzano & Lila & $51,19 \mathrm{f}-\mathrm{h}$ & $25,31 \mathrm{f}-\mathrm{i}$ & $59,78 \mathrm{j}-\mathrm{m}$ & $255 \mathrm{f}$ & $33,13 \mathrm{j}$ & $90,01 \mathrm{a}-\mathrm{g}$ & $8,10 \mathrm{~b}-\mathrm{i}$ & $1,48 \mathrm{~b}$ & 12,16 a-f & $62,81 \mathrm{ab}$ \\
\hline 24 & Negro 150 & $\begin{array}{l}\text { Negro opa- } \\
\text { co }\end{array}$ & 23,16 op & $0,36 \mathrm{n}$ & $267,80 \mathrm{~d}-\mathrm{f}$ & $250 \mathrm{~g}$ & $23,52 \mathrm{r}$ & $88,33 \mathrm{~d}-\mathrm{g}$ & $9,74 \mathrm{a}-\mathrm{g}$ & $1,78 \mathrm{~b}$ & $9,26 \mathrm{~d}-\mathrm{f}$ & $51,00 \mathrm{ab}$ \\
\hline 25 & Negro 8025 & $\begin{array}{l}\text { Negro opa- } \\
\text { co }\end{array}$ & 21,76 o-q & $0,82 \mathrm{n}$ & $265,20 d-f$ & $245 \mathrm{~h}$ & $17,32 \mathrm{w}$ & $87,69 \mathrm{~g}$ & 10,08 a-d & $2,00 \mathrm{~b}$ & $8,00 \mathrm{~d}-\mathrm{f}$ & $46,72 \mathrm{ab}$ \\
\hline
\end{tabular}

continúa... 
continuación Cuadro 2...

\begin{tabular}{|c|c|c|c|c|c|c|c|c|c|c|c|c|}
\hline \multirow{2}{*}{$\begin{array}{l}\text { Nú- } \\
\text { mero }\end{array}$} & \multirow[t]{2}{*}{ Cultivar } & \multicolumn{4}{|c|}{ Color } & \multicolumn{2}{|c|}{ Peso } & \multirow{2}{*}{$\begin{array}{c}\text { Cotile- } \\
\text { dón }(\%)\end{array}$} & \multirow{2}{*}{$\begin{array}{c}\text { Testa } \\
(\%)\end{array}$} & \multirow{2}{*}{$\begin{array}{l}\text { E,E, } \\
(\%)\end{array}$} & \multirow{2}{*}{$\begin{array}{l}\text { Cotile- } \\
\text { dón/Tes- } \\
\text { ta }\end{array}$} & \multirow{2}{*}{$\begin{array}{c}\text { Coti- } \\
\text { ledón/ } \\
\text { E,E, }\end{array}$} \\
\hline & & & $\begin{array}{l}\text { Lumino- } \\
\text { sidad }\left({ }^{\circ}\right)\end{array}$ & $\begin{array}{l}\text { Cromati- } \\
\text { cidad }\left({ }^{\circ}\right)\end{array}$ & Tono $\left({ }^{\circ}\right)$ & $\begin{array}{c}\text { Volu- } \\
\text { métrico } \\
(\mathrm{kg} / \mathrm{hl})\end{array}$ & $\begin{array}{c}100 \\
\text { semillas } \\
(\mathrm{g}) \\
\end{array}$ & & & & & \\
\hline 27 & $\begin{array}{l}\text { Negro Cha- } \\
\text { pingo }\end{array}$ & Negro & $17,85 \mathrm{r}$ & $0,42 \mathrm{n}$ & $\begin{array}{l}310,63 \\
a-c\end{array}$ & $240 \mathrm{i}$ & $18,19 \mathrm{v}$ & $88,39 \mathrm{~d}-\mathrm{g}$ & $9,81 \mathrm{a}-\mathrm{f}$ & $1,64 \mathrm{~b}$ & $9,47 \mathrm{c}-\mathrm{f}$ & $75,86 \mathrm{ab}$ \\
\hline 28 & $\begin{array}{l}\text { Negro Co- } \\
\text { taxtla }\end{array}$ & $\begin{array}{l}\text { Negro } \\
\text { opaco }\end{array}$ & $19,72 \mathrm{p}-\mathrm{r}$ & $0,92 \mathrm{n}$ & $261,33 \mathrm{~d}-\mathrm{f}$ & $250 \mathrm{~g}$ & $15,01 \mathrm{y}$ & $88,50 \mathrm{~d}-\mathrm{g}$ & 9,44 a-g & $1,95 \mathrm{~b}$ & $9,69 \mathrm{c}-\mathrm{f}$ & $46,13 a b$ \\
\hline 29 & $\begin{array}{l}\text { Negro Du- } \\
\text { rango }\end{array}$ & $\begin{array}{l}\text { Negro semi- } \\
\text { brillante }\end{array}$ & $27,78 \mathrm{n}$ & $0,76 n$ & $326,20 \mathrm{ab}$ & $260 \mathrm{e}$ & $32,90 \mathrm{jk}$ & 89,79 a-g & 8,60 a-i & $1,41 \mathrm{~b}$ & 11,08 a-f & $75,14 \mathrm{ab}$ \\
\hline 30 & $\begin{array}{l}\text { Negro } \\
\text { Huasteco } \\
81\end{array}$ & $\begin{array}{l}\text { Negro } \\
\text { opaco }\end{array}$ & 20,76 o-r & $0,55 \mathrm{n}$ & $134,45 \mathrm{~h}$ & $245 \mathrm{~h}$ & $17,00 \mathrm{w}$ & $88,35 \mathrm{~d}-\mathrm{g}$ & 9,93 a-e & $1,61 \mathrm{~b}$ & $9,14 \mathrm{~d}-\mathrm{f}$ & $59,24 \mathrm{ab}$ \\
\hline 31 & $\begin{array}{l}\text { Negro INI- } \\
\text { FAP }\end{array}$ & $\begin{array}{l}\text { Negro } \\
\text { opaco }\end{array}$ & $18,31 \mathrm{qr}$ & $0,62 \mathrm{n}$ & $272,73 \mathrm{c}-\mathrm{f}$ & $240 \mathrm{i}$ & $21,48 \mathrm{~s}$ & 91,10 a-e & $7,61 \mathrm{~d}-\mathrm{i}$ & $1,13 \mathrm{~b}$ & $12,54 \mathrm{a}-\mathrm{e}$ & $89,3 \mathrm{ab}$ \\
\hline 32 & $\begin{array}{l}\text { Negro Ja- } \\
\text { mapa }\end{array}$ & $\begin{array}{l}\text { Negro } \\
\text { opaco }\end{array}$ & 21,49 o-q & $0,37 \mathrm{n}$ & 130,66 h-i & $255 \mathrm{f}$ & $19,35 \mathrm{u}$ & 90,05 a-g & $7,69 \mathrm{~d}-\mathrm{i}$ & $1,88 \mathrm{~b}$ & 12,13 a-f & $56,41 \mathrm{ab}$ \\
\hline 33 & $\begin{array}{l}\text { Negro Me- } \\
\text { dellín }\end{array}$ & $\begin{array}{l}\text { Negro } \\
\text { opaco }\end{array}$ & 19,87 p-r & $0,33 \mathrm{n}$ & $199,43 \mathrm{~g}$ & $245 \mathrm{~h}$ & $18,11 \mathrm{v}$ & $90,01 \mathrm{a}-\mathrm{g}$ & $7,71 \mathrm{c}-\mathrm{i}$ & $2,00 \mathrm{~b}$ & $13,06 a-d$ & $47,70 \mathrm{ab}$ \\
\hline 34 & $\begin{array}{l}\text { Negro Na- } \\
\text { yarit }\end{array}$ & $\begin{array}{l}\text { Negro } \\
\text { opaco }\end{array}$ & $18,38 \mathrm{qr}$ & $1,03 n$ & $275,60 \mathrm{c}-\mathrm{f}$ & $250 \mathrm{~g}$ & 14,93 y & $88,92 \mathrm{c}-\mathrm{g}$ & $9,52 \mathrm{a}-\mathrm{g}$ & $1,47 \mathrm{~b}$ & $9,81 \mathrm{c}-\mathrm{f}$ & $65,15 a b$ \\
\hline 35 & $\begin{array}{l}\text { Negro } \\
\text { Otomí }\end{array}$ & $\begin{array}{l}\text { Negro } \\
\text { brillante }\end{array}$ & 19,80 p-r & $13,16 \mathrm{~m}$ & $329,80 \mathrm{a}$ & $250 \mathrm{~g}$ & 24,90 op & $88,17 \mathrm{fg}$ & 10,09 a-d & $1,69 \mathrm{~b}$ & 8,80 d-f & $55,37 \mathrm{ab}$ \\
\hline 36 & $\begin{array}{l}\text { Negro } \\
\text { Pacífico }\end{array}$ & $\begin{array}{l}\text { Negro } \\
\text { opaco }\end{array}$ & 20,50 o-r & $0,84 \mathrm{n}$ & $277,33 \mathrm{c}-\mathrm{f}$ & $245 \mathrm{~h}$ & $16,11 \mathrm{x}$ & $88,66 \mathrm{c}-\mathrm{g}$ & 9,644 a-g & $1,59 \mathrm{~b}$ & $9,25 \mathrm{~d}-\mathrm{f}$ & $58,07 \mathrm{ab}$ \\
\hline 37 & Negro Perla & $\begin{array}{l}\text { Negro bri- } \\
\text { llante }\end{array}$ & 21,34 o-r & $0,77 \mathrm{n}$ & 255,17 ef & $240 \mathrm{i}$ & $24,22 p-r$ & $84,77 \mathrm{~h}$ & $10,86 \mathrm{a}$ & $4,06 \mathrm{a}$ & $7,863 \mathrm{f}$ & $33,28 \mathrm{~b}$ \\
\hline 38 & $\begin{array}{l}\text { Negro } \\
\text { Sahuatoba }\end{array}$ & $\begin{array}{l}\text { Negro } \\
\text { opaco }\end{array}$ & 23,96 o & $0,68 \mathrm{n}$ & $\begin{array}{l}\begin{array}{l}301,20 \\
\text { a-d }\end{array} \\
\end{array}$ & $255 \mathrm{f}$ & $21,41 \mathrm{st}$ & $88,63 \mathrm{c}-\mathrm{g}$ & 9,66 a-g & $1,56 \mathrm{~b}$ & $9,58 \mathrm{c}-\mathrm{f}$ & $60,92 \mathrm{ab}$ \\
\hline 39 & $\begin{array}{l}\text { Negro } \\
\text { Sinaloa }\end{array}$ & $\begin{array}{l}\text { Negro } \\
\text { opaco }\end{array}$ & 20,62 o-r & $0,59 \mathrm{n}$ & $\begin{array}{l}287,13 \\
\text { b-e }\end{array}$ & $245 \mathrm{~h}$ & $14,60 \mathrm{y}$ & $89,75 \mathrm{a}-\mathrm{g}$ & 8,83 a-h & $1,35 \mathrm{~b}$ & $10,80 \mathrm{~b}-\mathrm{f}$ & $79,69 \mathrm{ab}$ \\
\hline 40 & $\begin{array}{l}\text { Negro } \\
\text { Tacaná }\end{array}$ & $\begin{array}{l}\text { Negro } \\
\text { opaco }\end{array}$ & 20,76 o-r & $0,54 \mathrm{n}$ & $265,90 \mathrm{~d}-\mathrm{f}$ & $240 \mathrm{i}$ & $18,70 \mathrm{uv}$ & $88,42 \mathrm{~d}-\mathrm{g}$ & 9,90 a-f & $1,49 \mathrm{~b}$ & $9,54 \mathrm{c}-\mathrm{f}$ & $61,68 \mathrm{ab}$ \\
\hline 41 & $\begin{array}{l}\text { Negro } \\
\text { Tropical }\end{array}$ & $\begin{array}{l}\text { Negro } \\
\text { opaco }\end{array}$ & $22,00 \mathrm{op}$ & $0,50 \mathrm{n}$ & $242,87 \mathrm{f}$ & $240 \mathrm{i}$ & $18,18 \mathrm{v}$ & $88,29 \mathrm{e}-\mathrm{g}$ & $10,13 \mathrm{a}-\mathrm{d}$ & $1,52 \mathrm{~b}$ & $9,17 \mathrm{~d}-\mathrm{f}$ & $97,81 \mathrm{ab}$ \\
\hline 42 & $\begin{array}{l}\text { Negro } \\
\text { Vizcaya }\end{array}$ & $\begin{array}{l}\text { Negro bri- } \\
\text { llante }\end{array}$ & 21,15 o-r & $1,10 \mathrm{n}$ & $322,50 \mathrm{ab}$ & $265 \mathrm{~d}$ & $34,47 \mathrm{i}$ & $88,98 \mathrm{c}-\mathrm{g}$ & $9,34 \mathrm{a}-\mathrm{g}$ & $1,45 \mathrm{~b}$ & $10,27 \mathrm{c}-\mathrm{f}$ & $64,45 \mathrm{ab}$ \\
\hline 43 & $\begin{array}{l}\text { NegroZaca- } \\
\text { tecas }\end{array}$ & $\begin{array}{l}\text { Negro bri- } \\
\text { llante }\end{array}$ & 22,95 op & $0,67 \mathrm{n}$ & $91,17 \mathrm{ij}$ & $260 \mathrm{e}$ & $20,64 \mathrm{t}$ & $87,66 \mathrm{gh}$ & $10,46 a b$ & $1,82 \mathrm{~b}$ & $8,53 \mathrm{ef}$ & $51,18 \mathrm{ab}$ \\
\hline 44 & $\begin{array}{l}\text { Ojo de Ca- } \\
\text { bra }\end{array}$ & $\begin{array}{l}\text { Pajizo/rayas } \\
\text { castaño }\end{array}$ & 47,52 i-1 & $34,57 \mathrm{c}-\mathrm{d}$ & $50,82 \mathrm{j}-\mathrm{m}$ & $74 \mathrm{n}$ & $32,32 \mathrm{k}$ & 90,21 a-g & $7,77 \mathrm{c}-\mathrm{i}$ & $1,57 \mathrm{~b}$ & $15,19 \mathrm{ab}$ & $61,06 \mathrm{ab}$ \\
\hline 45 & $\begin{array}{l}\text { Pinto An- } \\
\text { zalduas }\end{array}$ & $\begin{array}{l}\text { Gris variega- } \\
\text { do castaño }\end{array}$ & $46,98 \mathrm{j}-1$ & $24,93 \mathrm{f}-\mathrm{k}$ & $54,40 \mathrm{j}-\mathrm{m}$ & $270 \mathrm{c}$ & $25,55 \mathrm{o}$ & $88,92 \mathrm{c}-\mathrm{g}$ & 9,19 a-h & $1,76 \mathrm{~b}$ & $10,06 \mathrm{c}-\mathrm{f}$ & $52,68 \mathrm{ab}$ \\
\hline 46 & $\begin{array}{l}\text { Pinto Baya- } \\
\text { cora }\end{array}$ & $\begin{array}{l}\text { Pajizo va- } \\
\text { riegado cas- } \\
\text { taño }\end{array}$ & $46,95 \mathrm{j}-1$ & $23,94 \mathrm{f}-\mathrm{k}$ & $54,27 \mathrm{j}-\mathrm{m}$ & $265 \mathrm{~d}$ & $39,86 \mathrm{f}$ & $90,55 \mathrm{a}-\mathrm{g}$ & 7,50 e-i & $1,44 \mathrm{~b}$ & 12,65 a-e & $71,05 \mathrm{ab}$ \\
\hline 47 & $\begin{array}{l}\text { Pinto Mes- } \\
\text { tizo }\end{array}$ & $\begin{array}{l}\text { Pajizo va- } \\
\text { riegado cas- } \\
\text { taño }\end{array}$ & $43,01 \mathrm{~m}$ & $25,17 \mathrm{f}-\mathrm{i}$ & $54,60 \mathrm{j}-\mathrm{m}$ & $280 \mathrm{~b}$ & $44,74 \mathrm{c}$ & 89,04 b-g & $8,92 \mathrm{a}-\mathrm{h}$ & $1,97 \mathrm{~b}$ & $10,28 \mathrm{c}-\mathrm{f}$ & $69,48 \mathrm{ab}$ \\
\hline 48 & Pinto Villa & $\begin{array}{l}\text { Pajizo va- } \\
\text { riegado cas- } \\
\text { taño }\end{array}$ & $50,27 \mathrm{~g}-\mathrm{j}$ & $21,87 \mathrm{~g}-1$ & $59,97 \mathrm{j}-\mathrm{m}$ & $250 \mathrm{~g}$ & $39,84 \mathrm{fg}$ & 90,63 a-f & $7,84 \mathrm{c}-\mathrm{i}$ & $1,40 \mathrm{~b}$ & $12,10 \mathrm{a}-\mathrm{f}$ & $66,07 \mathrm{ab}$ \\
\hline
\end{tabular}

Valores seguidos por la misma letra dentro de columnas son estadísticamente similares $(\mathrm{P} \leq 0,05)$, 
ocho de las plántulas (biomasa del hipocótilo, folíolos y raíz, biomasa consumida durante la germinación y emergencia, diámetro del hipocótilo, longitud del hipocótilo y la raíz, y altura de la plántula) emergidas después de la siembra a 2,5, 5,0 y $10,0 \mathrm{~cm}$ de profundidad.

En el ACP los tres primeros componentes explicaron el 83,13\% de la variabilidad total observada, con 55,$03 ; 21,74$ y $6,36 \%$ en el primero, segundo y tercer $\mathrm{CP}$, respectivamente. Las variables de mayor contribución al CP1 fueron las ocho de las plántulas; en contraste, las de mayor contribución al CP2 fueron las cuatro de la semilla, y en el CP3 sólo el porcentaje de eje embrionario tuvo peso relativo elevado. La proporción de eje embrionario fue la única variable de las 12 incluidas en el análisis que destacó en dos CP (Cuadro 3). Este resultado destaca la importancia del eje embrionario en la variabilidad del proceso de germinación y emergencia en el germoplasma evaluado, y confirma lo señalado al respecto por Desai et al. (1997).

Cuadro 3. Vectores propios para los primeros tres componentes principales $(\mathrm{CP})$, derivados de cuatro atributos de semilla y ocho de plántula, de 48 cultivares de frijol mejorado en México, sembrados a tres profundidades. Montecillo, Texcoco, México. 2006.

\begin{tabular}{lrrr}
\hline Variable original & \multicolumn{3}{c}{ Vectores propios } \\
\cline { 2 - 4 } & CP1 & CP2 & CP3 \\
\hline Altura de la plántula (cm) & $\mathbf{0 , 9 5 3 4}$ & $-0,0421$ & $-0,0668$ \\
Biomasa de la raíz (g) & $\mathbf{0 , 8 7 8 6}$ & 0,0510 & 0,2090 \\
Biomasa del hipocótilo (g) & $\mathbf{0 , 9 0 2 6}$ & 0,0757 & $-0,0198$ \\
Biomasa de los folíolos (g) & $\mathbf{0 , 8 7 9 5}$ & 0,0229 & 0,0737 \\
Biomasa consumida (\%) & $\mathbf{0 , 9 4 8 4}$ & $-0,0911$ & $-0,1283$ \\
Cotiledón (porcentaje de la & & & \\
semilla) & $-0,0134$ & $\mathbf{0 , 9 6 0 7}$ & $-0,0051$ \\
Diámetro del hipocótilo (cm) & $\mathbf{0 , 9 5 5 7}$ & 0,0072 & $-0,0776$ \\
Eje embrionario (porcentaje & & & \\
de la semilla) & 0,0316 & $\mathbf{- 0 , 6 5 7 2}$ & $\mathbf{0 , 7 1 1 6}$ \\
Longitud de hipocótilo (cm) & $\mathbf{0 , 8 6 4 2}$ & $-0,0732$ & $-0,1136$ \\
Longitud de raíz (cm) & $\mathbf{0 , 8 7 2 7}$ & $-0,0230$ & 0,0727 \\
Peso de 100 semillas (g) & 0,1064 & $\mathbf{0 , 6 7 7 0}$ & 0,2966 \\
Testa (porcentaje de la se- & $-0,0346$ & $\mathbf{- 0 , 8 7 8 4}$ & $-0,2737$ \\
milla) & & & \\
\hline
\end{tabular}

Los valores en negritas son las variables de mayor contribución al los componentes principales, $\mathrm{CP} 1, \mathrm{CP} 2$ y CP3.
La ordenación con respecto al CP1 y CP2 mostró la polarización de dos grandes conjuntos de cultivares y la disposición marginal de los cultivares Núm. 2 ('Azufrado Higuera') y 37 ('Negro Perla') fuera del resto de la dispersión provocada por el CP2. La ordenación sobre los CP1 y CP3 confirmó el patrón de concentraciones extremas sobre el CP1 de las entidades biológicas relacionadas con la siembra a 2,5 y 5,0 $\mathrm{cm}$ de profundidad, y la separación del cultivar Núm. 37, provocada por el CP3 (Figura 1). El conjunto más numeroso, de los dos separados por el CP1, en ambas


Figura 1. Ordenación mediante el análisis de componentes principales $\mathrm{CP} 1$ y CP2 y $\mathrm{CP} 1$ y $\mathrm{CP} 3$, de 48 cultivares de frijol mejorado, sembradas a tres profundidades (a: $2,5 \mathrm{~cm}, \mathrm{~b}: 5,0 \mathrm{~cm}$ y c: $10,0 \mathrm{~cm}$ ), basada en cuatro atributos morfológicos de la semilla, y ocho atributos de las plántulas. Montecillo, Texcoco, México. 2006. 
ordenaciones está conformado principalmente por entidades biológicas relacionadas con la siembra a 2,5 y $5,0 \mathrm{~cm}$ de profundidad (número de cultivar seguido por las letra a o b, respectivamente), aunque, también contiene algunas relacionadas con la siembra a $10 \mathrm{~cm}$ de profundidad. En contraste, el conjunto compacto en los cuadrantes del lado izquierdo, está conformado casi exclusivamente por entidades biológicas relacionadas con 28 cultivares cuyas plántulas no emergieron cuando fueron sembrados a $10 \mathrm{~cm}$ de profundidad (Figura 1).

Estos resultados son una muestra de la variabilidad en un atributo vital, la emergencia de los cultivares mejorados, en dependencia de la profundidad de siembra. De acuerdo con los resultados del ACP, para el $99 \%$ de los cultivares fue indistinto que se sembrara a 2,5 ó 5,0 cm, y también para cerca del $60 \%$ cuando se sembraron a $10 \mathrm{~cm}$ de profundidad. Estos resultados también muestran que la expresión del vigor de la semilla en la germinación y emergencia es dependiente de la profundidad de siembra. Además, puede decirse que los resultados evidencian la variabilidad reducida en el vigor seminal entre los cultivares mejorados. Sin embargo, el análisis de varianza y la comparación múltiple de medias mostró diferencias estadísticas significativas en siete de las ocho características de las plántulas generadas con las tres profundidades de siembra (Cuadro 4). Al respecto, la altura de la plántula, la biomasa acumulada en los folíolos e hipocótilo, y el diámetro de este último en las plántulas de la siembra a $5 \mathrm{~cm}$ de profundidad, en promedio para los 48 cultivares corresponden a plántulas más robustas que cuando se sembraron a 2,5 y $10 \mathrm{~cm}$. En contraste, la altura significativamente menor de las plántulas, el crecimiento limitado de la raíz y acumulación reducida de biomasa en los folíolos, incipientemente desarrollados, de las plántulas de la siembra a $10 \mathrm{~cm}$ de profundidad, indican que estas plántulas fueron las menos vigorosas de los tres grupos con profundidad de siembra distinta (Cuadro 4). Los cultivares ordenados a lo largo del $\mathrm{CP} 1$, entre los valores $-0,14$ y 1,0 , se ordenaron con base en un gradiente de vigor de las plántulas. Así, los localizados entre los valores $-0,14$ y 0,2 , como los Núm. 7, 26, 31 y 42 generaron plántulas pequeñas (con 12 a $19 \mathrm{~cm}$ de altura), con raíces cortas (entre 3 y 7,9 $\mathrm{cm}$ ) y acumulación de biomasa reducida en la raíz (entre 0,03 y $0,05 \mathrm{~g}$ ) y en las hojas (entre 0,05 y $0,15 \mathrm{~g}$ ) con la siembra a $10 \mathrm{~cm}$ de profundidad; mientras que, en el extremo derecho del CP1, entre los valores 0,70 y 0,85, como los cultivares Núm. 4, 5, 6, 9, 12,16, 21, 23, 27 y 37 generaron las plántulas más vigorosas y altas (entre 24 y $32 \mathrm{~cm}$ ), con las raíces más extensas (de 9 a $17 \mathrm{~cm})$ y acumulación mayor de biomasa en la raíz (de 0,07 a $0,17 \mathrm{~g}$ ) y las hojas (de 0,20 a $0,36 \mathrm{~g}$ ) con la siembra a 2,5 y $5,0 \mathrm{~cm}$ de profundidad (Figura 1 y Cuadro 4). Cabe destacar que el promedio de la biomasa residual de los cotiledones (o al contrario, el porcentaje de biomasa consumida), después de la emergencia fue estadísticamente igual para todos los cultivares con las tres profundidades de siembra (Cuadro 4). Esto indica que la mayor proporción de las reservas de la semilla, localizadas en los cotiledones, en promedio fueron consumidas en proporciones similares durante la germinación y emergencia de las plántulas, independientemente de su asignación heterogénea a las estructuras de las plántulas, como reacción a las diferencias en la profundidad de siembra.

Los resultados de este estudio coinciden con los obtenidos en trigo (Triticum turgidum L.) por Mahdi et al. (1998); estos autores, concluyeron que el vigor de

Cuadro 4. Efecto de la profundidad de siembra sobre las características de las plántulas de 48 cultivares mejorados en México. Montecillo, Texcoco, México. 2006.

\begin{tabular}{|c|c|c|c|c|c|c|c|c|}
\hline \multirow{2}{*}{$\begin{array}{c}\text { Profundidad } \\
\text { de siembra } \\
(\mathrm{cm})\end{array}$} & \multirow{2}{*}{$\begin{array}{l}\text { Altura de } \\
\text { la plántula } \\
(\mathbf{c m})\end{array}$} & \multicolumn{4}{|c|}{ Biomasa } & \multicolumn{2}{|c|}{ Hipocótilo (cm) } & \multirow{2}{*}{$\begin{array}{c}\text { Longitud } \\
\text { de raíz } \\
(\mathrm{cm})\end{array}$} \\
\hline & & $\begin{array}{c}\text { Folíolos } \\
\text { (g) }\end{array}$ & $\begin{array}{c}\text { Hipocótilo } \\
\text { (g) }\end{array}$ & $\begin{array}{c}\text { Raíz } \\
\text { (g) }\end{array}$ & $\begin{array}{c}\text { Consumida } \\
(\%)\end{array}$ & Diámetro & Longitud & \\
\hline 2,5 & $22,34 \mathrm{~b}$ & 0,196 a & $0,052 \mathrm{~b}$ & 0,075 a & 90,85 a & $0,363 \mathrm{c}$ & $6,00 \mathrm{c}$ & 12,14 a \\
\hline 5,0 & 23,91 a & 0,195 a & 0,062 a & $0,070 \mathrm{~b}$ & 90,40 a & 0,435 a & $9,38 \mathrm{~b}$ & $10,37 \mathrm{~b}$ \\
\hline 10,0 & $20,76 \mathrm{c}$ & $0,139 \mathrm{~b}$ & 0,064 a & $0,055 \mathrm{c}$ & 90,89 a & $0,413 \mathrm{~b}$ & $9,81 \mathrm{a}$ & $7,74 \mathrm{c}$ \\
\hline
\end{tabular}

Valores seguidos por la misma letra dentro de columnas son estadísticamente similares $(\mathrm{P} \leq 0,05)$.

Los valores en negritas son las variables de los tratamientos con valores significativamente más altos. 
la semilla disminuye con la profundidad de siembra, ya que a $12 \mathrm{~cm}$ se generaron brotes delgados y más largos que con la siembra a 3 y $6 \mathrm{~cm}$ de profundidad. Kirby (1993) señaló que el incremento de la profundidad de siembra reduce la tasa de crecimiento del primordio, por lo tanto la tasa de emergencia, el número total de hojas en el tallo principal, la elongación del tallo y la longitud de los entrenudos. Soltani et al. (2006) señalaron que el retardo de la emergencia puede reducir la cantidad de plántulas que emergen, pues se incrementa la oportunidad de que la semilla y la plántula sean invadidas por patógenos del suelo. Algo similar pudo haber sucedido en el frijol sembrado a 10 $\mathrm{cm}$ de profundidad en el presente estudio, pues la tasa de emergencia y la emergencia total con la siembra a $10 \mathrm{~cm}$ de profundidad se redujo significativamente (resultados no mostrados).

La mayoría de los cultivares ordenados en el cuadrante superior derecho, como los Núm. 2, 4, 5, 7 a 10, 14, 22, 23, 31, 32, 44, y 46 de la Figura 1, se caracterizan por su proporción significativamente alta de cotiledón, proporción de testa significativamente menor, o ambas (Cuadro 2). En contraste los cultivares Núm. 1, 15, 19, 20, 24 a 28, 36, 40 y 41, ubicados en el cuadrante inferior derecho de la misma Figura 1 son parte del grupo que posee las proporciones mayores de testa del grupo evaluado y tiene semillas pequeñas o ligeras (Cuadro 2).

El tamaño de la semilla también puede ser una característica relevante para asegurar el éxito de la emergencia. Leishman et al. (2000) señalaron que las reservas de la semilla son translocadas a las diferentes estructuras de la plántula en forma progresiva, y en algún periodo durante este proceso de translocación el tamaño de semilla debe tener ventajas, pues una semilla grande permitirá incrementar o asegurar la cantidad suficiente de reservas que la plántula requiera para concluir su desarrollo, inclusive en condiciones adversas.

\section{Análisis de agrupamiento de $\mathbf{4 8}$ cultivares, basado en las características seminales y de las plántulas}

La clasificación de los cultivares con base en cuatro características de las semillas (i.e. peso de 100 semillas, y proporción de cotiledones, eje embrionario y testa) y ocho de las plántulas (biomasa del hipocótilo, folíolos, raíz y consumida durante la germinación y emergencia, diámetro del hipocótilo, longitud del hipocótilo y la raíz, y altura de la plántula), con coeficiente de correlación cofenética de 0,8 , parte de la conformación de dos conjuntos principales, A y B. La diferencia entre éstos es que el primero incluyó a los cultivares que originaron plántulas emergidas con la siembra a 2,5, 5,0 y $10 \mathrm{~cm}$, mientras que el conjunto B conformó un solo grupo con los que no emergieron (Figura 2).

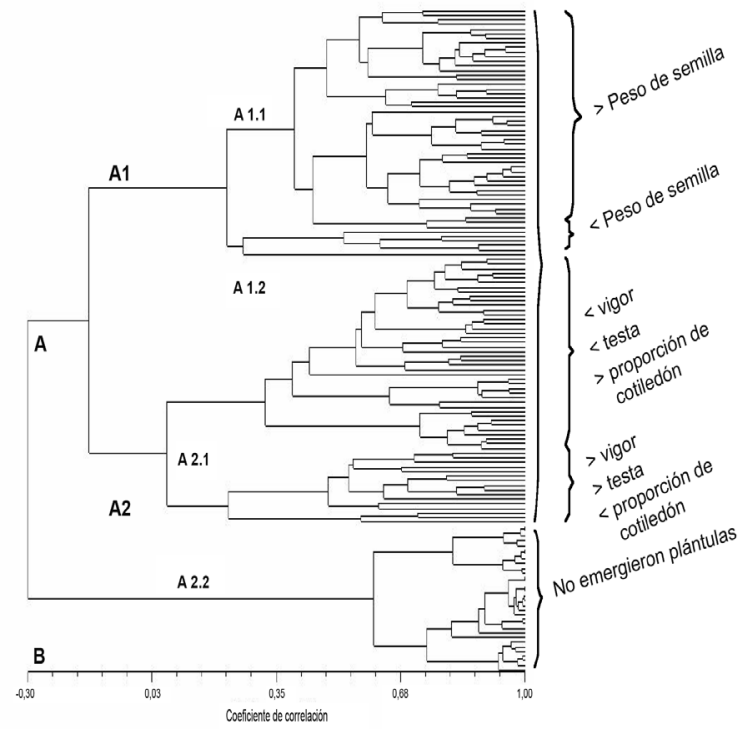

Figura 2. Clasificación de cultivares de frijol mejorado con base en atributos de sus semillas y vigor inicial, sembrados a tres profundidades $(2,5,5,0$ y 10,0 cm), Montecillo, Texcoco, México. 2006.

El conjunto A se dividió en dos subconjuntos (A.1 y A.2); a la vez, cada uno de ellos se dividió en dos grupos. El grupo A1.1 presentó consumo de reservas estadísticamente similar al conjunto A1.2 (90,10 y 90,31\%), a pesar de la diferencia significativa, de $6,15 \mathrm{~g}$, en el peso de 100 semillas entre ambos (Cuadros 5 y 6; Figura 2).

En contraste con el subconjunto A1, ambos grupos de subconjuntos A2 incluyeron semillas significativamente más pesadas, pero de estos dos, el grupo A2.1 presentó proporción mayor de cotiledón y menor de testa (Cuadro 5). Aunque, el consumo de reservas del grupo A2.1 fue estadísticamente igual al grupo 
Cuadro 5. Características de las semillas de cinco grupos de cultivares de frijol (Phaseolus vulgaris L.) formados por el análisis de conglomerados, basado en cuatro atributos seminales de 48 cultivares de frijol mejorado. Texcoco, México. 2006.

\begin{tabular}{lcccc}
\hline Grupo & $\begin{array}{c}\text { Peso de 100 } \\
\text { semillas }(\mathbf{g})\end{array}$ & $\begin{array}{c}\text { Cotiledón } \\
(\boldsymbol{\%})\end{array}$ & $\begin{array}{c}\text { Testa } \\
(\boldsymbol{\%})\end{array}$ & $\begin{array}{c}\text { Eje em- } \\
\text { brionario } \\
(\boldsymbol{\%})\end{array}$ \\
\hline A1.1 & $20,92 \mathrm{e}$ & $88,33 \mathrm{~d}$ & $9,73 \mathrm{a}$ & $1,81 \mathrm{a}$ \\
$\mathrm{A} 1.2$ & $27,05 \mathrm{~d}$ & $89,01 \mathrm{c}$ & $9,24 \mathrm{~b}$ & $1,53 \mathrm{~b}$ \\
$\mathrm{~A} 2.1$ & $33,56 \mathrm{~b}$ & $90,69 \mathrm{a}$ & $7,62 \mathrm{~d}$ & $1,51 \mathrm{~b}$ \\
A2.2 & $38,88 \mathrm{a}$ & $89,10 \mathrm{c}$ & $9,33 \mathrm{~b}$ & $1,47 \mathrm{~b}$ \\
$\mathrm{~B}$ & $28,12 \mathrm{c}$ & $89,49 \mathrm{~b}$ & $8,83 \mathrm{c}$ & $1,59 \mathrm{a}$ \\
\hline
\end{tabular}

Valores seguidos por la misma letra dentro de columnas son estadísticamente similares $(\mathrm{P} \leq 0,05)$.

A2.2 (Cuadro 6), las plántulas desarrolladas de semillas pesadas (A2.2) fueron en promedio más vigorosas, pues tuvieron mayor altura y diámetro de hipocótilo, y acumularon más biomasa en su raíz y folíolos (Cuadro 6). En contraste, las semillas más ligeras o pequeñas, las incluidas en el grupo A1.1, generaron algunas de las plántulas más cortas, con los folíolos, hipocótilo y raíz más ligeros, e hipocótilo angosto y corto (Cuadros 5 у 6 ).
El grupo B incluyó los 28 cultivares cuyas plántulas no emergieron cuando su semilla fue sembrada a $10 \mathrm{~cm}$ de profundidad, más otros cuatro que tampoco lo lograron con la siembra a $5 \mathrm{~cm}$ (Figura 2). Debido a que las condiciones de siembra, emergencia y desarrollo de la plántula fueron similares para todos los cultivares, es posible suponer que la emergencia fue dependiente del cultivar y la profundidad de siembra. Los cultivares cuyas plántulas no emergieron, tienen en promedio, semillas con tamaño intermedio, con proporciones también intermedias de cotiledón, testa y eje embrionario en la semilla, respecto a las que sí generaron plántulas emergidas (Cuadro 5); es decir, entre las características seminales ninguna sobresalió o pudo ser relacionada con la incapacidad de los cultivares para emerger. Es muy probable que las semillas de estos cultivares hayan germinado, pero que a la vez hayan sido incapaces de emerger, al agotar sus reservas por su metabolismo menos eficiente. Allende-Arrarás et al. (2006) señalaron la existencia de una asociación positiva entre el tamaño de la semilla, la capacidad de absorción de agua y el rendimiento, e indicaron que las semillas grandes absorben más agua que las pequeñas. Así, también es posible que este grupo de semillas no tuviera una capacidad adecuada de absorción de agua para iniciar los procesos metabólicos de la germinación. Gontia y Awasthi (1999) señalaron que la emergencia y desarrollo de las plántulas de semillas grandes es mayor, y que éstas luego tienen mejor desarrollo vegetativo y reproductivo.

Cuadro 6. Características de los cinco grupos de cultivares de frijol (Phaseolus vulgaris L.) mejorado, formados por el análisis de conglomerados basado en ocho atributos de las plántulas emergidas de siembras a 2,5; 5,0 y $10,0 \mathrm{~cm}$ de profundidad. Montecillo, Texcoco, México. 2006.

\begin{tabular}{|c|c|c|c|c|c|c|c|c|}
\hline \multirow[t]{2}{*}{ Grupo } & \multirow{2}{*}{$\begin{array}{l}\text { Altura de la } \\
\text { plántula (cm) }\end{array}$} & \multicolumn{4}{|c|}{ Biomasa } & \multicolumn{2}{|c|}{ Hipocótilo (cm) } & \multirow{2}{*}{$\begin{array}{l}\text { Longitud de } \\
\text { raíz }(\mathrm{cm})\end{array}$} \\
\hline & & $\begin{array}{c}\text { Folíolos } \\
\text { (g) }\end{array}$ & $\begin{array}{l}\text { Hipocótilo } \\
\text { (g) }\end{array}$ & $\begin{array}{l}\text { Raíz } \\
\text { (g) }\end{array}$ & $\begin{array}{c}\text { Consumida } \\
(\%)\end{array}$ & Diámetro & Longitud & \\
\hline A1.1 & $22,35 \mathrm{~b}$ & $0,169 \mathrm{~b}$ & $0,048 \mathrm{c}$ & $0,062 \mathrm{~b}$ & $90,10 \mathrm{c}$ & $0,38 \mathrm{c}$ & $7,94 \mathrm{c}$ & $10,02 \mathrm{c}$ \\
\hline A 1.2 & $21,46 \mathrm{c}$ & $0,192 \mathrm{a}$ & $0,092 \mathrm{a}$ & $0,059 \mathrm{~b}$ & $90,31 \mathrm{bc}$ & $0,42 \mathrm{~b}$ & $9,00 \mathrm{a}$ & $12,92 \mathrm{a}$ \\
\hline A 2.1 & $22,62 b$ & 0,198 a & $0,062 \mathrm{~b}$ & $0,076 \mathrm{a}$ & $91,27 \mathrm{a}$ & $0,41 \mathrm{~b}$ & $7,82 \mathrm{c}$ & $11,17 \mathrm{~b}$ \\
\hline A 2.2 & $24,24 \mathrm{a}$ & $0,200 \mathrm{a}$ & $0,062 \mathrm{~b}$ & $0,076 \mathrm{a}$ & $91,04 \mathrm{ab}$ & $0,43 \mathrm{a}$ & $8,33 \mathrm{~b}$ & $10,41 \mathrm{c}$ \\
\hline B & $0,00 \mathrm{~d}$ & $0,000 \mathrm{c}$ & $0,000 \mathrm{~d}$ & $0,000 \mathrm{c}$ & - & $0,00 \mathrm{~d}$ & $0,0 \mathrm{~d}$ & $0,00 \mathrm{~d}$ \\
\hline
\end{tabular}

Valores seguidos por la misma letra dentro de columnas son estadísticamente similares $(\mathrm{P} \leq 0,05)$. 


\section{Acervos y razas}

Respecto a la predominancia de cultivares de las diferentes razas reconocidas actualmente, Singh (1999) señaló que los cultivares de las razas Durango, Mesoamérica (ambas mesoamericanas), y Nueva Granada, con origen andino, ocupan más que $80 \%$ del área mundial cultivada con frijol; además, los cultivares de la raza Jalisco, también mesoamericanos, son cultivados exclusivamente en los valles altos de México. Así las razas Durango (36 \%), Mesoamérica $(29 \%)$ y Jalisco (29\%), del almacén genético mesoamericano, representaron $93 \%$ de los cultivares incluidos en el presente estudio (Cuadro 1). Es conveniente señalar que Sonnante et al. (1994) y Singh (2001) aseguran que la variación genética dentro de los cultivares comerciales de frijol es estrecha, por lo que es común sus niveles bajos de tolerancia a diversos factores causantes de estrés.

El análisis estadístico confirmó la diferencia de tamaño en la semilla de los dos acervos (andino y mesoamericano, Cuadro 7), la cual solo había sido apreciada por Singh (2001) y Voysest (2000), pues los cultivares del acervo mesoamericano representó en promedio sólo $63 \%$ del acervo andino. Además, se encontró que las semillas del acervo andino parecen contener una proporción significativamente mayor de reservas (los cotiledones representan más que 90 $\%$ del peso de la semilla). Entre los cultivares de la raza Mesoamérica se presentó además un gradiente de peso de semilla y proporción de cotiledones; así, las semillas de peso menor, presentaron proporción significativamente mayor de eje embrionario y testa (Cuadro 7).

En promedio, las semillas pesadas del acervo andino generaron plántulas más vigorosas, con más biomasa en folíolos, hipocótilo y raíz, respecto a las del acervo mesoamericano; sin embargo, la altura de las plántulas de la raza Durango fue estadísticamente igual a las de la raza Nueva Granada, y el consumo promedio de reservas fue estadísticamente igual entre las razas Durango, Jalisco y Nueva Granada (Cuadro 8).

Con la siembra a $10 \mathrm{~cm}$ de profundidad los cultivares del acervo de Mesoamérica presentaron capacidad de emergencia y establecimiento de plántulas significativamente mayor (44,30\%) que el acervo andino $(33,33 \%)$. Esta diferencia parece sin relación con el tamaño medio de la semilla de cada acervo, pues como en el caso de la raza Nueva Granada, 33,33 \% de los cultivares de la raza Mesoamérica emergieron con la siembra a $10,0 \mathrm{~cm}$, aunque su peso promedio seminal fuera menos que la mitad respecto al de Nueva Granada. Sin embargo, dentro del acervo mesoamericano sí

Cuadro 7. Características de las semillas de 48 cultivares de frijol (Phaseolus vulgaris L,) mejorado, de cuatro razas de los acervos Andino y Mesoamericano, Montecillo, Texcoco, México. 2006.

\begin{tabular}{lcccc}
\hline \multicolumn{1}{c}{ Acervo } & $\begin{array}{c}\text { Peso de 100 } \\
\text { semillas } \mathbf{( g )}\end{array}$ & $\begin{array}{c}\text { Cotiledón* } \\
(\boldsymbol{\%})\end{array}$ & $\begin{array}{c}\text { Eje embrionario* } \\
(\boldsymbol{\%})\end{array}$ & $\begin{array}{c}\text { Testa* } \\
(\boldsymbol{\%})\end{array}$ \\
\hline Andino & $42,54 \mathrm{a}$ & $91,08 \mathrm{a}$ & $1,41 \mathrm{~b}$ & $7,56 \mathrm{~b}$ \\
Mesoamericano & $26,66 \mathrm{~b}$ & $89,20 \mathrm{~b}$ & $1,67 \mathrm{a}$ & $8,98 \mathrm{a}$ \\
Raza & & & & \\
Durango & $34,39 \mathrm{~b}$ & $89,60 \mathrm{~b}$ & $8,72 \mathrm{c}$ & $1,53 \mathrm{c}$ \\
Jalisco & $25,48 \mathrm{c}$ & $89,16 \mathrm{c}$ & $9,04 \mathrm{~b}$ & $1,67 \mathrm{~b}$ \\
Mesoamerica & $18,18 \mathrm{~d}$ & $88,74 \mathrm{~d}$ & $9,23 \mathrm{a}$ & $1,83 \mathrm{a}$ \\
Nueva Granada & $42,54 \mathrm{a}$ & $91,08 \mathrm{a}$ & $7,56 \mathrm{~d}$ & $1,41 \mathrm{c}$ \\
\hline
\end{tabular}

Valores seguidos por la misma letra dentro de columnas y acervo o raza son estadísticamente similares $(\mathrm{P} \leq 0,05)$.

*Proporción de la materia seca seminal. 
Cuadro 8. Características de las plántulas de 48 cultivares de frijol (Phaseolus vulgaris L.) mejorado en México, de cuatro razas (Durango, Jalisco, Mesoamérica y Nueva Granada) de los acervos Andino y Mesoamericano, Montecillo, Texcoco, México, 2006.

\begin{tabular}{|c|c|c|c|c|c|c|c|c|}
\hline \multirow[t]{2}{*}{ Acervos } & \multirow{2}{*}{$\begin{array}{l}\text { Altura de } \\
\text { la plántula } \\
\text { (cm) }\end{array}$} & \multicolumn{4}{|c|}{ Biomasa* } & \multicolumn{2}{|c|}{ Hipocótilo (cm) } & \multirow{2}{*}{$\begin{array}{l}\text { Longitud de } \\
\text { raíz }(\mathrm{cm})\end{array}$} \\
\hline & & $\begin{array}{c}\text { Folíolos } \\
\text { (g) }\end{array}$ & $\begin{array}{c}\text { Hipocótilo } \\
\text { (g) }\end{array}$ & Raíz (g) & $\begin{array}{c}\text { Consumida } \\
(\%)\end{array}$ & Diámetro & Longitud & \\
\hline Andino & 23,14 a & 0,1843 a & 0,0620 a & 0,0961 a & 91,19 a & 0,42 a & $7,56 \mathrm{~b}$ & 11,18 a \\
\hline Mesoamericano & $22,69 \mathrm{~b}$ & $0,1545 \mathrm{~b}$ & $0,0582 \mathrm{~b}$ & $0,0675 \mathrm{~b}$ & $90,60 \mathrm{~b}$ & $0,40 \mathrm{~b}$ & 8,11 a & $10,69 \mathrm{~b}$ \\
\hline \multicolumn{9}{|l|}{ Raza } \\
\hline Durango & 23,76 a & $0,1630 \mathrm{~b}$ & 0,0646 a & $0,0717 \mathrm{~b}$ & $90,65 \mathrm{ab}$ & $0,41 \mathrm{~b}$ & 8,26 a & $10,58 \mathrm{~b}$ \\
\hline Jalisco & $21,75 \mathrm{~b}$ & $0,1345 \mathrm{c}$ & $0,0555 \mathrm{~b}$ & $0,0622 \mathrm{c}$ & $90,76 \mathrm{ab}$ & $0,40 \mathrm{~b}$ & $7,89 \mathrm{~b}$ & $10,60 \mathrm{~b}$ \\
\hline Mesoamérica & $21,81 \mathrm{~b}$ & $0,1628 \mathrm{~b}$ & $0,0517 \mathrm{~b}$ & $\begin{array}{c}0,0668 \\
\mathrm{bc}\end{array}$ & $90,35 \mathrm{~b}$ & $0,39 \mathrm{c}$ & 8,13 a & $10,95 \mathrm{ab}$ \\
\hline Nueva Granada & 23,14 a & 0,1843 a & 0,0620 a & 0,0961 a & 91,19 a & 0,42 a & $7,56 \mathrm{c}$ & 11,18 a \\
\hline
\end{tabular}

Valores seguidos por la misma letra dentro de columnas y acervo o raza son estadísticamente similares $(\mathrm{P} \leq 0,05)$.

Los valores en negritas son las variables de los tratamientos con valores significativamente más altos.

*Expresada en materia seca.

se presentó un gradiente de emergencia con la siembra a $10,0 \mathrm{~cm}$, relacionado con el peso promedio de la semilla, con 33,33; 46,15 y 53,33\% de emergencia para los cultivares de la raza Mesoamérica (las semillas más ligeras de todas), Jalisco (intermedias) y Durango (las más pesadas; Cuadro 7), respectivamente.

\section{CONCLUSIONES}

La variabilidad de la morfología seminal de una muestra de 48 cultivares de frijol mejorados en México para diferentes regiones agrícolas, es amplia si se consideran caracteres como el peso de la semilla, parámetros del color (cromaticidad, luminosidad y tono) de la testa y razas; pero la variabilidad del vigor inicial de los 48 cultivares, dependiente de la profundidad de siembra es reducido. Se demostró la utilidad de los análisis multivariables, tanto de ordenación como de agrupamiento, para identificar semejanzas y diferencias morfológicas de las semillas y las plántulas del germoplasma mejorado, con origen andino y mesoamericano.

\section{LITERATURA CITADA}

Allende-Arrarás, G; Acero, GM; Padilla, RJ; Mayek, PN. 2006. Comportamiento agronómico y características físico-químicas del grano del frijol en Aguascalientes, México. Revista Fitotecnia Mexicana 29(1): 89-93.

Beebe, S; Skroch, PW; Tohme, J; Duque, MC; Pedraza, F; Nienhuis, J. 2000. Structure of genetic diversity among common bean landraces of Middle American origin based on correspondence analysis of RAPD. Crop Science 40: 264-273.

Cruz, R. 2006. Crean súper frijol: produce su fertilizante y resiste tres semanas sin agua. Boletín semanal Núm. 31: Granos Básicos 3 p.

Debouck, DG; Toro, O; Paredes, OM; Jonson, WC; Gepts, P. 1993. Genetic diversity and ecological distribution of Phaseolus vulgaris (Fabaceae) in northwestern South America. Economic Botany 47: 408-423.

Desai, BB; Kotecha, PM; Salunkhe, DK. 1997. Seeds handbook. Marcel Dekker, New Cork, U.S.A. 627 p. 
Duffus, CM; Slaughter, JC. 1992. Seeds and their uses. Wiley. New York, NY, USA. 154 p.

Flores, DA. 2002. Potencial agroindustrial del frijol silvestre (Phaseolus vulgaris L.): Composición química. Tesis de licenciatura, Departamento de Agroindustrias, Universidad Autónoma Chapingo. 75 p.

Franco, TL; Hidalgo, R. 2003. Análisis estadístico de datos de caracterización morfológica de recursos fitogenéticos. Boletín técnico Núm. 8. Instituto Internacional de Recursos Fitogenéticos, Cali, Colombia. 89 p.

Gontia, SA; Awasthi, MK. 1999. Effect of seed grading by size on various seed vigour attributes, morpho-physiological characters and seed yield in soybean (Glycine $\max ($ L.) Merrill) genotypes. Seed Research 27(1): 25-30.

Hidalgo, R. 2003. Variabilidad genética y caracterización de especies vegetales en análisis estadístico de datos de caracterización morfológica de recursos fitogenéticos. Boletín técnico Núm. 8. Instituto Internacional de Recursos Fitogenéticos (IPGRI), Cali, Colombia. 89 p.

Kaplan, L; Lynch, TF. 1999. Phaseolus (Fabaceae) in archaeology: AMS radiocarbon dates and their significance for pre-Colombian agriculture. Economic Botany 53(3): 261-272.

Kirby, JM. 1993. Effect of sowing depth on seedling emergence, growth and development in barley and wheat. Field Crop Research 35: 101-111.

Leishman, MR; Wright, IJ; Moles, AT; Westoby, M. 2000. The evolutionary ecology of seed size. In: Seeds: The ecology of regeneration in plant communities. Fenner, M. (ed.). 2 edition. CAB International. p. 31-57.

Mahdi, L; Bell, CJ; Ryan, J. 1998. Establishment and yield of wheat (Triticum turgidum L.) after early sowing at various depths in a semi-arid Mediterranean environment. Field Crop Research 58:187-196.

Moreno, ME. 1996. Análisis físico y biológico de semillas agrícolas. Programa Universitario de Alimentos. UNAM. México. 393 p.
Núñez, C; Barrientos, PA. 2006. Estimación de la variabilidad interna de muestras poblacionales, mediante análisis de componentes principales. Interciencia 31(11): 802-806.

Otálora, JM; Ligarreto, GA; Romero, A. 2006. Comportamiento de frijol común (Phaseolus vulgaris L.) tipo reventón por características agronómicas y de calidad de grano. Agronomía Colombiana 24(1): 7-16.

Peña-Valdivia, CB; Aguirre, JR. 2007. El frijol silvestre mexicano (Phaseolus vulgaris L.): Riqueza renovable desaprovechada. Universidad Autónoma de San Luis Potosí. 150 p. En prensa.

Peña-Valdivia, CB; Aguirre, JR; García, E. Del R.; Muruaga, JS. 1998. Componentes del rendimiento de una semilla de una población silvestre y un cultivar de frijol (Phaseolus vulgaris L.). Quaderni di Botanica Ambientale e Applicata 6: 181-187.

Revilla, P; Butrón, A; Malvar, RA; Ordás, A. 1999. Relationships among kernel weight, early vigor, and growth in maize. Crop Science 39: 654-658.

Rosales, RS; Acosta-Gallegos, JA; Durán, RP; Guillén, H; Pérez, P; Esquivel, G; Muruaga, JS. 2003. Diversidad genética del germoplasma mejorado de frijol (Phaseolus vulgaris L.) en México. Agricultura Técnica en México 29(1): 11-24.

Singh, SP; Gepts, P; Debouck, DG. 1991. Races of common bean (Phaseolus vulgaris, Fabaceae). Economic Botany 45 (3): 379-396.

Singh, S. P. 1999. Production and utilization. In: S.P. SINGH (ed.) Common bean improvement in the twenty-first century. p. 1-24

Singh, S.P. 2001. Broadening the genetic base of common bean cultivars. Crop Science 41: 1659-1675.

Soltani, A; Robertson, MJ; Torabi, B; Yousefi-Daz, M; Sarparast, R. 2006. Modelling seedling emergence in chickpea as influences by temperature and sowing depth. Agricultural and Forest Meteorology 138: 156167. 
Sonnante, G; Stockton, T; Nodari, RO; Becerra-Velásquez, VL; Gepts, P. 1994. Evolution of genetic diversity during domestication of common-bean (Phaseolus vulgaris L.). Theoretical Applied Genetic 89: 629-635.

Vargas, VML; Muruaga, JS; J. Acosta G; Navarrete, R; Pérez, P; Esquivel, G; Irizar, MBG; Hernández, JM. 2006. Colección Núcleo de Phaseolus vulgaris L. del INIFAP. Libro técnico 10. Secretaría de Agricultura, Ganadería, Desarrollo Rural, Pesca y Alimentación. 461 p.
Vidal-Barahona, A; Lagunes-Espinoza, LC; Valadez, E; Ortiz-García, CF. 2006. Variabilidad morfológica y molecular de cultivares criollos y mejorados de frijol común en Tabasco, México. Revista Fitotecnia Mexicana 29(4): 273-281.

Voysest, VO. 2000. Mejoramiento genético del frijol (Phaseolus vulgaris L.): legado de variedades de América Latina 1930-1999. Centro Internacional de Agricultura Tropical. Cali, Colombia. 195 p. 